\title{
Identification of a GGAG motif in the HIV-2 5' leader RNA that is critical for genome dimerisation and viral replication Anne L'Hernault*, Jane Greatorex and Andrew Lever
}

\author{
Address: Department of Medicine, University of Cambridge, Cambridge CB2 0QQ, UK
}

* Corresponding author

from Frontiers of Retrovirology: Complex retroviruses, retroelements and their hosts

Montpellier, France. 2I-23 September 2009

Published: 24 September 2009

Retrovirology 2009, 6(Suppl 2):P49 doi:10.1 I86/1742-4690-6-S2-P49

This abstract is available from: http://www.retrovirology.com/content/6/S2/P49

(C) 2009 L'Hernault et al; licensee BioMed Central Ltd.

\section{Background}

The RNA packaging signal (Psi) of Human Immunodeficiency Virus type-2 (HIV-2) is located upstream of the splice donor and hence is present on both spliced and unspliced RNAs. Encapsidation specificity is conferred by a co-translational mechanism and limited availability of the Gag protein. Specific structures within the defined Psi region have been proposed to influence RNA packaging and viral replication, in particular the formation of an extended stem-loop 1 (SL-1). In addition, we have demonstrated previously that the viral RNA genome is dimeric within the virion particles and that motifs within Psi are critical for this process. Yet, little is known regarding the sequence and structural elements required for HIV-2 genome dimerisation and the exact relationship between genome dimerisation and encapsidation, as well as how these two processes relate to HIV-2 infectivity, remains unclear.

\section{Materials and methods}

In this study, we designed a series of viral mutants aimed at investigating RNA motifs within the Psi region and SL1 structure. Wild type (WT) and mutant viruses were evaluated for their ability to infect and replicate in T-cells. Evolution studies of the viruses were performed and potential revertants investigated. Furthermore, the genomes of WT and mutant HIV-2 were analysed by native northern blot and their packaging efficiencies were determined.

\section{Results}

Contrary to previous observations, we show that the formation of stem B is not required for RNA encapsidation or viral replication. However, purine-rich sequences, and in particular a GGAG motif at position 392-395 of the HIV2 ROD genome, appear to be important for viral infectivity. Furthermore, our results confirm the existence of a tight relationship between dimerisation and encapsidation of HIV-2 genomic RNA. Mutant viruses that failed to dimerise also showed a packaging defect. However, the reverse is not true, suggesting that genome dimerisation is important for encapsidation and that dimer formation likely occurs prior to packaging. Our results also imply that a non-reciprocal relationship exists between the two processes.

\section{Conclusion}

In HIV-2, there is a correlation between genome dimerisation, packaging and viral replication and the purine-rich motif GGAG at position 392-395 is essential for the establishment of a productive infection. 